\title{
Syzygies, multigraded regularity and toric varieties
}

\author{
Milena Hering, Hal Schenck and Gregory G. Smith
}

\begin{abstract}
Using multigraded Castelnuovo-Mumford regularity, we study the equations defining a projective embedding of a variety $X$. Given globally generated line bundles $B_{1}, \ldots, B_{\ell}$ on $X$ and $m_{1}, \ldots, m_{\ell} \in \mathbb{N}$, consider the line bundle $L:=B_{1}^{m_{1}} \otimes \cdots \otimes B_{\ell}^{m_{\ell}}$. We give conditions on the $m_{i}$ which guarantee that the ideal of $X$ in $\mathbb{P}\left(H^{0}(X, L)^{*}\right)$ is generated by quadrics and that the first $p$ syzygies are linear. This yields new results on the syzygies of toric varieties and the normality of polytopes.
\end{abstract}

\section{Introduction}

Understanding the equations that cut out a projective variety $X$ and the syzygies among them is a central problem in algebraic geometry. To give precise statements, consider the morphism $\varphi_{L}: X \rightarrow \mathbb{P}\left(H^{0}(X, L)^{*}\right)$ induced by a globally generated line bundle $L$ on $X$. Let $S=\operatorname{Sym}^{\bullet} H^{0}(X, L)$ be the homogeneous coordinate ring of $\mathbb{P}\left(H^{0}(X, L)^{*}\right)$, and let $E_{\bullet}$ be a minimal free graded resolution of the graded $S$-module $R=\bigoplus_{j \geqslant 0} H^{0}\left(X, L^{j}\right)$ associated to $L$. Following [GL85], we say that $L$ satisfies $\left(N_{p}\right)$ for $p \in \mathbb{N}$ provided that $E_{0} \cong S$ and $E_{i}=\bigoplus S(-i-1)$ for all $1 \leqslant i \leqslant p$. Thus, $\varphi_{L}(X)$ is projectively normal if and only if $L$ satisfies $\left(N_{0}\right)$ and $\varphi_{L}(X)$ is normal. If $L$ satisfies $\left(N_{1}\right)$, then the homogeneous ideal of $\varphi_{L}(X)$ is generated by quadrics and $\left(N_{2}\right)$ implies that the relations among the generators are linear. In [Mum70], properties $\left(N_{0}\right)$ and $\left(N_{1}\right)$ are called normal generation and normal presentation, respectively.

Although it was shown in [Gre84b] that any sufficiently ample line bundle on an arbitrary variety satisfies $\left(N_{p}\right)$, it is normally difficult to determine which multiple of a given ample line bundle suffices. When $X$ is a smooth curve of genus $g$, it was proved in [Gre84a] that a line bundle $L$ of degree at least $2 g+1+p$ satisfies $\left(N_{p}\right)$. This is recovered from an analogous statement for finite sets in [GL88]. When $X$ is a smooth variety of dimension $n$ and $L$ is very ample, it was shown in [EL93] that the adjoint line bundles of the form $K_{X}+(n+1+p) L$ satisfy $\left(N_{p}\right)$. Explicit criteria for $\left(N_{p}\right)$ are also given in [GP99, GP01] for surfaces and in [Par00, PP04] for abelian varieties; we refer to $[\mathrm{Laz} 04, \S 1.8$.D] for a survey. The primary goal of this paper is to produce similar conditions for toric varieties.

To achieve this, we use multigraded Castelnuovo-Mumford regularity. Fix a list $B_{1}, \ldots, B_{\ell}$ of globally generated line bundles on $X$. For $\boldsymbol{u}=\left(u_{1}, \ldots, u_{\ell}\right) \in \mathbb{Z}^{\ell}$, set $B^{\boldsymbol{u}}:=B_{1}^{u_{1}} \otimes \cdots \otimes B_{\ell}^{u_{\ell}}$ and let $\mathcal{B}$ be the semigroup $\left\{B^{\boldsymbol{u}}: \boldsymbol{u} \in \mathbb{N}^{\ell}\right\} \subset \operatorname{Pic}(X)$. We say that a line bundle $L$ is $\mathscr{O}_{X}$-regular (with respect to $\left.B_{1}, \ldots, B_{\ell}\right)$ if $H^{i}\left(X, L \otimes B^{-\boldsymbol{u}}\right)=0$ for all $i>0$ and all $\boldsymbol{u} \in \mathbb{N}^{\ell}$ with $|\boldsymbol{u}|:=u_{1}+\cdots+u_{\ell}=i$. Our main technical result is the following.

Theorem 1.1. Let $\boldsymbol{w}_{1}, \boldsymbol{w}_{2}, \boldsymbol{w}_{3}, \ldots$ be a sequence in $\mathbb{N}^{\ell}$ such that $B^{\boldsymbol{w}_{i}} \otimes B_{j}^{-1} \in \mathcal{B}$ for $1 \leqslant j \leqslant \ell$ and set $\boldsymbol{m}_{i}:=\boldsymbol{w}_{1}+\cdots+\boldsymbol{w}_{i}$ for $i \geqslant 1$. If $B^{\boldsymbol{m}_{1}}$ is $\mathscr{O}_{X}$-regular, then $B^{\boldsymbol{m}_{p}}$ satisfies $\left(N_{p}\right)$ for $p \geqslant 1$.

Received 4 March 2005, accepted in final form 2 August 2006.

2000 Mathematics Subject Classification 14M25 (primary), 13D02, 14C20, 52B20 (secondary).

Keywords: toric variety, syzygy, Castelnuovo-Mumford regularity.

This journal is (C) Foundation Compositio Mathematica 2006. 


\section{Hering, H. Schenck And G. G. Smith}

The case $\ell=1$ is Theorem 1.3 in [GP99]. Our proof is a multigraded variant of the arguments in [GP99]. Applying Theorem 1.1 with $\ell=1$ to line bundles on toric varieties yields the following.

Corollary 1.2. Let $L$ be an ample line bundle on an $n$-dimensional toric variety. If $d \geqslant n-1+p$, then the line bundle $L^{d}$ satisfies $\left(N_{p}\right)$.

The case $p=0$, an ingredient in our proof, was established in [EW91]; other proofs appear in [BGT97, LTZ93, ON02]. On a toric surface, it was proved in [Koe93] that $L$ satisfies $\left(N_{1}\right)$ if the associated lattice polygon contains more than three lattice points in its boundary. In [GP01], a criterion was given for $\left(N_{p}\right)$ on smooth rational surfaces which, when restricted to toric surfaces, shows that $L$ satisfies $\left(N_{p}\right)$ if the associated polygon contains at least $p+3$ lattice points in its boundary. This result extends to all toric surfaces and is sharp; see [Sch04]. Related results on toric surfaces appear in [Fak02] which studies multiplication maps and in [Har97] which studies $\left(N_{0}\right)$ for anticanonical rational surfaces. For an arbitrary toric variety, it was shown in [BGT97] that $R$ is Koszul when $d \geqslant n$ and this implies that $L^{d}$ satisfies $\left(N_{1}\right)$ when $d \geqslant n$. Assuming $n \geqslant 3$, Ogata established in [Oga03] that $L^{n-1}$ satisfies $\left(N_{1}\right)$ and, building on this in [Oga04], he proved that $L^{n-2+p}$ satisfies $\left(N_{p}\right)$ when $n \geqslant 3$ and $p \geqslant 1$.

We can strengthen Corollary 1.2 by using additional invariants. Let

$$
h_{L}(d):=\chi\left(L^{d}\right)=\sum_{i=0}^{n}(-1)^{i} \operatorname{dim} H^{i}\left(X, L^{d}\right)
$$

be the Hilbert polynomial of $L$ and let $r(L)$ be the number of integer roots of $h_{L}$.

Corollary 1.3. Let $L$ be a globally generated line bundle on a toric variety and let $r(L)$ be the number of integer roots of its Hilbert polynomial $h_{L}$. If $p \geqslant 1$ and $d \geqslant \max \left\{\operatorname{deg}\left(h_{L}\right)-r(L)+p-1, p\right\}$, then the line bundle $L^{d}$ satisfies $\left(N_{p}\right)$.

If $X=\mathbb{P}^{n}$ and $L=\mathscr{O}_{X}(1)$, then we have $h_{L}(d)=\left(\begin{array}{c}d+n \\ n\end{array}\right)$ and $r(L)=n$. In particular, we recover [Gre84b, Theorem 2.2] which states that $\mathscr{O}_{\mathbb{P}^{n}}(d)$ satisfies $\left(N_{p}\right)$ for $p \leqslant d$. On the other hand, [OP01, Theorem 2.1] shows that, for $n \geqslant 2$ and $d \geqslant 3, \mathscr{O}_{\mathbb{P}^{n}}(d)$ does not satisfy $\left(N_{3 d-2}\right)$ and it is conjectured that this is sharp.

Using the dictionary between lattice polytopes and line bundles on toric varieties, Corollary 1.3 yields a normality criterion for lattice polytopes. A lattice polytope $P$ is normal if every lattice point in $m P$ for $m \geqslant 1$ is a sum of $m$ lattice points in $P$. Let $r(P)$ be the largest integer such that $r(P) P$ does not contain any lattice points in its interior.

Corollary 1.4. If $P$ is a lattice polytope of dimension $n$, then $(n-r(P)) P$ is normal.

Theorem 1.1 also applies to syzygies of Segre-Veronese embeddings.

Corollary 1.5. If $X=\mathbb{P}^{n_{1}} \times \cdots \times \mathbb{P}^{n_{\ell}}$, then $\mathscr{O}_{X}\left(d_{1}, \ldots, d_{\ell}\right)$ satisfies $\left(N_{p}\right)$ for $p \leqslant \min \left\{d_{i}: d_{i} \neq 0\right\}$.

The Segre embedding $\mathscr{O}_{X}(1, \ldots, 1)$ satisfies $\left(N_{p}\right)$ if and only if $p \leqslant 3$; see [Las78, PW85] for $\ell=2$, and [Rub02, Rub04] for $\ell>2$. An overview of results and conjectures about the syzygies of Segre-Veronese embeddings appears in [EGHP06, §3].

Inspired by [EL93], we also examine the syzygies of adjoint bundles. Recall that a line bundle on a toric variety is numerically effective (nef) if and only if it is globally generated, and the dualizing sheaf $K_{X}$ is a line bundle if and only if $X$ is Gorenstein.

Corollary 1.6. Let $X$ be a projective $n$-dimensional Gorenstein toric variety and let $B_{1}, \ldots, B_{\ell}$ be the minimal generators of the Nef cone $\operatorname{Nef}(X)$. Suppose that $\boldsymbol{w}_{1}, \boldsymbol{w}_{2}, \ldots$ is a sequence in $\mathbb{N}^{\ell}$ such that $B^{\boldsymbol{w}_{i}} \otimes B_{j}^{-1} \in \mathcal{B}$ for $1 \leqslant j \leqslant \ell$ and $\boldsymbol{m}_{i}:=\boldsymbol{w}_{1}+\cdots+\boldsymbol{w}_{i}$ for $i \geqslant 1$. If $X \neq \mathbb{P}^{n}$ and $p \geqslant 1$, 


\section{SyZYGIES, MULTIGRADED REGULARITY AND TORIC VARIETIES}

then the adjoint line bundle $K_{X} \otimes B^{\boldsymbol{m}_{n+p}}$ satisfies $\left(N_{p}\right)$. If $X=\mathbb{P}^{n}$ and $p \geqslant 1$, then $K_{X} \otimes B^{\boldsymbol{m}_{n+1+p}}$ satisfies $\left(N_{p}\right)$.

In [EL93], it was proved that for a very ample line bundle $L$ and a nef line bundle $N$ on a smooth $n$-dimensional algebraic variety $X \neq \mathbb{P}^{n}, K_{X} \otimes L^{n+p} \otimes N$ satisfies $\left(N_{p}\right)$. Corollary 1.6 gives a similar result for ample line bundles on possibly singular Gorenstein toric varieties. Specifically, if $L$ is an ample line bundle such that $L \otimes B_{j}^{-1} \in \mathcal{B}$ for $1 \leqslant j \leqslant \ell$ and $N$ is a nef line bundle on $X \neq \mathbb{P}^{n}$ then $K_{X} \otimes L^{n+p} \otimes N$ satisfies $\left(N_{p}\right)$. For an ample line bundle $L$ on a ruled variety $X, \S 5$ in [But94] demonstrates that $K_{X} \otimes L^{n+1+p}$ need not satisfy $\left(N_{p}\right)$ for $p=0$ and 1. Hence, the conclusions of Corollary 1.6 are stronger than one can expect for a general variety. The proof of Corollary 1.6 combines Theorem 1.1 with Fujita's freeness conjecture for toric varieties, see [Fuj03].

Conventions. We work over a field of characteristic zero and $\mathbb{N}$ denotes the nonnegative integers.

\section{Multigraded Castelnuovo-Mumford regularity}

This section reviews multigraded regularity as introduced in [MS04]. Fix a list $B_{1}, \ldots, B_{\ell}$ of globally generated line bundles on $X$. For $\boldsymbol{u}:=\left(u_{1}, \ldots, u_{\ell}\right) \in \mathbb{Z}^{\ell}$, set $B^{\boldsymbol{u}}:=B_{1}^{u_{1}} \otimes \cdots \otimes B_{\ell}^{u_{\ell}}$ and let $\mathcal{B}$ be the semigroup $\left\{B^{\boldsymbol{u}}: \boldsymbol{u} \in \mathbb{N}^{\ell}\right\} \subset \operatorname{Pic}(X)$. If $\boldsymbol{e}_{1}, \ldots, \boldsymbol{e}_{\ell}$ is the standard basis for $\mathbb{Z}^{\ell}$, then $B^{\boldsymbol{e}_{j}}=B_{j}$.

Let $\mathscr{F}$ be a coherent $\mathscr{O}_{X}$-module and let $L$ be a line bundle on $X$. We say that $\mathscr{F}$ is $L$-regular (with respect to $\left.B_{1}, \ldots, B_{\ell}\right)$ provided that $H^{i}\left(X, \mathscr{F} \otimes L \otimes B^{-\boldsymbol{u}}\right)=0$ for all $i>0$ and all $\boldsymbol{u} \in \mathbb{N}^{\ell}$ satisfying $|\boldsymbol{u}|:=u_{1}+\cdots+u_{\ell}=i$. When $X=\mathbb{P}^{n}$, this definition specializes to Mumford's version of regularity (see [Mum66]) and as Mumford says, 'this apparently silly definition reveals itself as follows'.

TheOREm 2.1. If the coherent sheaf $\mathscr{F}$ is $L$-regular, then for all $\boldsymbol{u} \in \mathbb{N}^{\ell}$ :

(1) $\mathscr{F}$ is $\left(L \otimes B^{\boldsymbol{u}}\right)$-regular;

(2) the map $H^{0}\left(X, \mathscr{F} \otimes L \otimes B^{\boldsymbol{u}}\right) \otimes H^{0}\left(X, B^{\boldsymbol{v}}\right) \rightarrow H^{0}\left(X, \mathscr{F} \otimes L \otimes B^{\boldsymbol{u}+\boldsymbol{v}}\right)$ is surjective for all $\boldsymbol{v} \in \mathbb{N}^{\ell}$;

(3) $\mathscr{F} \otimes L \otimes B^{\boldsymbol{u}}$ is globally generated provided that there exists $\boldsymbol{w} \in \mathbb{N}^{\ell}$ such that $B^{\boldsymbol{w}}$ is ample.

When $X$ is a toric variety, this follows from results in $[\mathrm{MS} 04, \S 6]$. Our approach imitates the proofs of Theorem 2 in [Mum70] and Proposition II.1.1. in [Kle66].

Proof. By replacing $\mathscr{F}$ with $\mathscr{F} \otimes L$, we may assume that the coherent sheaf $\mathscr{F}$ is $\mathscr{O}_{X}$-regular. We proceed by induction on $\operatorname{dim}(\operatorname{Supp}(\mathscr{F}))$. The claim is trivial when $\operatorname{dim}(\operatorname{Supp}(\mathscr{F})) \leqslant 0$. As each $B_{j}$ is basepoint-free, we may choose a section $s_{j} \in H^{0}\left(X, B_{j}\right)$ such that the induced map $\mathscr{F} \otimes B^{-\boldsymbol{e}_{j}} \rightarrow \mathscr{F}$ is injective (see [Mum70, p. 43]). If $\mathscr{G}_{j}$ is the cokernel of this map, then we have $0 \rightarrow \mathscr{F} \otimes B^{-\boldsymbol{e}_{j}} \rightarrow \mathscr{F} \rightarrow \mathscr{G}_{j} \rightarrow 0$ and $\operatorname{dim}\left(\operatorname{Supp}\left(\mathscr{G}_{j}\right)\right)<\operatorname{dim}(\operatorname{Supp}(\mathscr{F}))$. From this short exact sequence, we obtain the long exact sequence

$$
\rightarrow H^{i}\left(X, \mathscr{F} \otimes B^{-\boldsymbol{u}-\boldsymbol{e}_{j}}\right) \rightarrow H^{i}\left(X, \mathscr{F} \otimes B^{-\boldsymbol{u}}\right) \rightarrow H^{i}\left(X, \mathscr{G}_{j} \otimes B^{-\boldsymbol{u}}\right) \rightarrow H^{i+1}\left(X, \mathscr{F} \otimes B^{-\boldsymbol{u}-\boldsymbol{e}_{j}}\right) \rightarrow
$$

By taking $|\boldsymbol{u}|=i$, we deduce that $\mathscr{G}_{j}$ is $\mathscr{O}_{X}$-regular. The induction hypothesis implies that $\mathscr{G}_{j}$ is $\left(B_{j}\right)$-regular. Setting $\boldsymbol{u}=-\boldsymbol{e}_{j}+\boldsymbol{u}^{\prime}$ with $\left|\boldsymbol{u}^{\prime}\right|=i$, we see that $\mathscr{F}$ is $\left(B_{j}\right)$-regular and part (1) follows.

For part (2), consider the following commutative diagram.

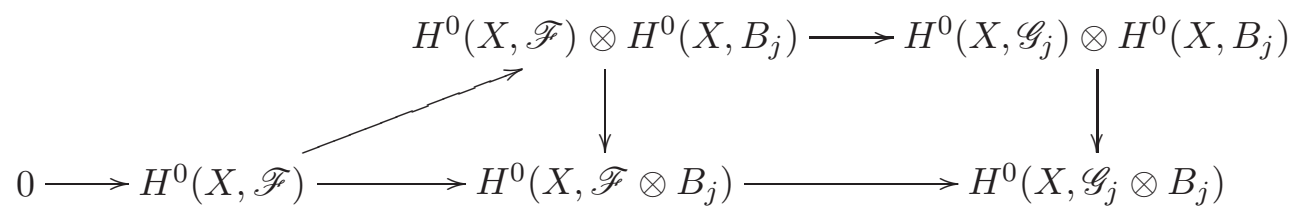




\section{Hering, H. Schenck And G. G. Smith}

Since $\mathscr{F}$ is $\mathscr{O}_{X}$-regular, the map in the top row is surjective. The induction hypothesis guarantees that the map in the right column is surjective. Thus, the Snake lemma (see, e.g., [Bou80, Proposition 1.2]) implies that the map in the middle column is also surjective. Therefore, part (2) follows from the associativity of the tensor product and part (1).

Finally, consider the following commutative diagram.

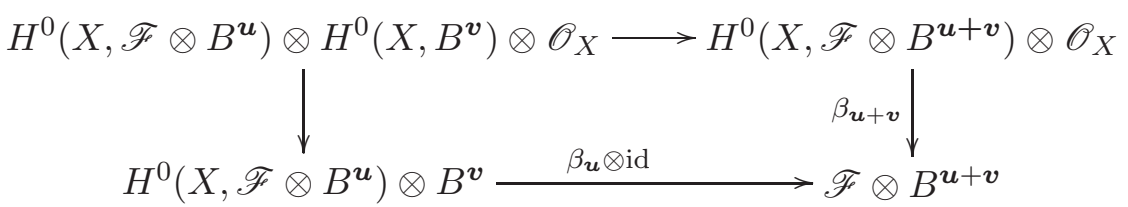

Applying part (2), we see that the map in the top row is surjective. By assumption, there is $\boldsymbol{w} \in \mathbb{N}^{\ell}$ such that $B^{\boldsymbol{w}}$ is ample. If $\boldsymbol{v}:=k \boldsymbol{w}$, then Serre's vanishing theorem (see, e.g., [Laz04, Theorem 1.2.6]) implies that $\beta_{\boldsymbol{u}+\boldsymbol{v}}$ is surjective for $k \gg 0$. Hence, $\beta_{\boldsymbol{u}}$ is also surjective which proves part (3).

We end this section with an elementary observation.

Lemma 2.2. Let $0 \rightarrow \mathscr{F}^{\prime} \rightarrow \mathscr{F} \rightarrow \mathscr{F}^{\prime \prime} \rightarrow 0$ be a short exact sequence of coherent $\mathscr{O}_{X}$-modules. If $\mathscr{F}$ is L-regular, $H^{0}\left(X, \mathscr{F} \otimes L \otimes B^{-\boldsymbol{e}_{j}}\right) \rightarrow H^{0}\left(X, \mathscr{F}^{\prime \prime} \otimes L \otimes B^{-\boldsymbol{e}_{j}}\right)$ is surjective for all $1 \leqslant j \leqslant \ell$, and $\mathscr{F}^{\prime \prime}$ is $\left(L \otimes B^{-\boldsymbol{e}_{j}}\right)$-regular for all $1 \leqslant j \leqslant \ell$, then $\mathscr{F}^{\prime}$ is also $L$-regular.

Sketch of the Proof. Tensor the exact sequence $0 \rightarrow \mathscr{F}^{\prime} \rightarrow \mathscr{F} \rightarrow \mathscr{F}^{\prime \prime} \rightarrow 0$ with $L \otimes B^{\boldsymbol{u}}$ and analyze the associated long exact sequence. The argument is similar to the proof of Theorem 2.1(1).

\section{Proof of the main theorem}

The proof of Theorem 1.1 combines multigraded Castelnuovo-Mumford regularity with a cohomological criterion for $\left(N_{p}\right)$. Given a globally generated line bundle $L$ on $X$, there is a natural surjective map $\operatorname{ev}_{L}: H^{0}(X, L) \otimes \mathscr{O}_{X} \rightarrow L$ and we set $M_{L}:=\operatorname{Ker}\left(\operatorname{ev}_{L}\right)$. Hence, $M_{L}$ is a vector bundle on $X$ which sits in the short exact sequence

$$
0 \rightarrow M_{L} \rightarrow H^{0}(X, L) \otimes \mathscr{O}_{X} \rightarrow L \rightarrow 0 .
$$

It is well known that $M_{L}$ governs the syzygies of $\varphi_{L}(X)$ in $\mathbb{P}\left(H^{0}(X, L)^{*}\right)$. Specifically, $L$ satisfies $\left(N_{p}\right)$ if and only if $H^{1}\left(X, \bigwedge^{q} M_{L} \otimes L^{j}\right)=0$ for $q \leqslant p+1$ and $j \geqslant 1$; see [GL88, Lemma 1.10] or [Laz89, Proposition 1.3.3]. In characteristic zero, $\bigwedge^{k} M_{L}$ is a direct summand of $M_{L}^{\otimes k}$, so it suffices to show that $H^{1}\left(X, M_{L}^{\otimes q} \otimes L^{j}\right)=0$ for $q \leqslant p+1$ and $j \geqslant 1$ in our situation.

Proof of Theorem 1.1. Set $L:=B^{m_{p}}$ and let $M_{L}$ be the vector bundle in (†). We first prove, by induction on $q$, that $M_{L}^{\otimes q}$ is $\left(B^{m_{q}}\right)$-regular for all $q \geqslant 1$. Since $B^{m_{1}}$ is $\mathscr{O}_{X^{-}}$-regular, Theorem 2.1(2) implies that $H^{0}\left(X, B^{\boldsymbol{m}_{1}+\boldsymbol{u}}\right) \otimes H^{0}\left(X, B^{\boldsymbol{v}}\right) \rightarrow H^{0}\left(X, B^{\boldsymbol{m}_{1}+\boldsymbol{u}+\boldsymbol{v}}\right)$ is surjective for all $\boldsymbol{u}, \boldsymbol{v} \in \mathbb{N}^{\ell}$. In particular, the maps $H^{0}(X, L) \otimes H^{0}\left(X, B^{\boldsymbol{m}_{1}-\boldsymbol{e}_{j}}\right) \rightarrow H^{0}\left(X, L \otimes B^{\boldsymbol{m}_{1}-\boldsymbol{e}_{j}}\right)$ for $1 \leqslant j \leqslant \ell$ are surjective because $B^{m_{1}} \in \bigcap_{j=1}^{\ell}\left(B_{j} \otimes \mathcal{B}\right)$. Combining Theorem 2.1(1) and Lemma 2.2, we see that $M_{L}$ is $\left(B^{\boldsymbol{m}_{1}}\right)$-regular. For $q>1$, tensor the sequence $(\dagger)$ with $M_{L}^{\otimes(q-1)}$ to obtain the exact sequence $0 \rightarrow M_{L}^{\otimes q} \rightarrow H^{0}(X, L) \otimes M_{L}^{\otimes(q-1)} \rightarrow M_{L}^{\otimes(q-1)} \otimes L \rightarrow 0$. The induction hypothesis states that $M_{L}^{\otimes(q-1)}$ is $\left(B^{\boldsymbol{m}_{q-1}}\right)$-regular. Since $B^{\boldsymbol{w}_{q}} \otimes B_{j}^{-1} \in \mathcal{B}$ for all $1 \leqslant j \leqslant \ell$, Theorem 2.1(2) shows that $H^{0}\left(X, M_{L}^{\otimes(q-1)} \otimes B^{\boldsymbol{m}_{q}-\boldsymbol{e}_{j}}\right) \otimes H^{0}(X, L) \rightarrow H^{0}\left(X, M_{L}^{\otimes(q-1)} \otimes L \otimes B^{\boldsymbol{m}_{q}-\boldsymbol{e}_{j}}\right)$ is surjective for $1 \leqslant j \leqslant \ell$. Again by Theorem 2.1(1) and Lemma $2.2, M_{L}^{q}$ is $\left(B^{m_{q}}\right)$-regular.

As observed above, it suffices to prove that $H^{1}\left(X, M_{L}^{\otimes q} \otimes L^{j}\right)=0$ for $q \leqslant p+1$ and $j \geqslant 1$. Since $M_{L}^{\otimes q}$ is $\left(B^{m_{q}}\right)$-regular, Theorem $2.1(1)$ implies that $M_{L}^{\otimes q}$ is $\left(B^{m_{p}}\right)$-regular for $1 \leqslant q \leqslant p$; 


\section{SyZYGIES, MULTIGRADED REGULARITY AND TORIC VARIETIES}

as $\mathscr{O}_{X}$ is $\left(B^{m_{1}}\right)$-regular, Theorem $2.1(1)$ also implies that $\mathscr{O}_{X}$ is $\left(B^{m_{p}}\right)$-regular. It follows that $H^{1}\left(X, M_{L}^{\otimes q} \otimes L^{j}\right)=0$ for $q \leqslant p$ and $j \geqslant 1$. Moreover, Theorem 2.1(2) shows that

$$
H^{0}(X, L) \otimes H^{0}\left(X, M_{L}^{\otimes p} \otimes L^{j}\right) \rightarrow H^{0}\left(X, M_{L}^{\otimes p} \otimes L^{j+1}\right)
$$

is surjective and the exact sequence $0 \rightarrow M_{L}^{\otimes(p+1)} \otimes L^{j} \rightarrow H^{0}(X, L) \otimes M_{L}^{\otimes p} \otimes L^{j} \rightarrow M_{L}^{\otimes p} \otimes L^{j+1} \rightarrow 0$ implies that $H^{1}\left(X, M_{L}^{\otimes(p+1)} \otimes L^{j}\right)=0$ for $j \geqslant 1$.

\section{Applications to toric varieties}

In this section, we apply the main theorem to line bundles on an $n$-dimensional projective toric variety $X$. Consider a globally generated line bundle $L$ on $X$ and its associated lattice polytope $P_{L}$. Let $r(L)$ be the number of integer roots of the Hilbert polynomial $h_{L}(d)=\chi\left(L^{d}\right)$. Since the higher cohomology of $L^{d}$ vanishes and the lattice points in the polytope $d P_{L}=P_{L^{d}}$ form a basis for $H^{0}\left(X, L^{d}\right)$, it follows that $h_{L}(d)$ is equal to the Ehrhart polynomial of $P_{L}$. In other words, $h_{L}(d)$ is the number of lattice points in $d P$. If $r\left(P_{L}\right)$ is the largest integer such that $r\left(P_{L}\right) P_{L}$ does not contain any interior lattice points, then Ehrhart reciprocity (see, e.g., [Sta97, Corollary 4.6.28]) implies that the integer roots of $h_{L}(d)$ are $\left\{-1, \ldots,-r\left(P_{L}\right)\right\}$ and $r\left(P_{L}\right)=r(L)$.

Lemma 4.1. If $L$ is a globally generated line bundle on a toric variety $X$ and $r(L)$ is the number of integer roots of its Hilbert polynomial $h_{L}$, then $L^{\operatorname{deg}\left(h_{L}\right)-r(L)}$ is $\mathscr{O}_{X}$-regular with respect to $L$.

Proof. We must establish that $H^{i}\left(X, L^{\operatorname{deg}\left(h_{L}\right)-r(L)-i}\right)=0$ for all $i>0$. If $\operatorname{deg}\left(h_{L}\right)-r(L)-i \geqslant 0$, this follows from the vanishing of the higher cohomology of globally generated line bundles on a complete toric variety; see [Ful93, §3.5]. When $\operatorname{deg}\left(h_{L}\right)-r(L)-i<0$, we follow the proof of Theorem 2.5 in [BB96]. Let $X^{\prime}$ be the toric variety corresponding to the normal fan to $P_{L}$. There is a canonical toric map $\psi: X \rightarrow X^{\prime}$ and an ample line bundle $A$ on $X^{\prime}$ such that $H^{i}\left(X, L^{r}\right) \cong H^{i}\left(X^{\prime}, A^{r}\right)$ for all $r \in \mathbb{Z}$. A toric version of the Kodaira vanishing theorem establishes that $H^{j}\left(X, L^{-u}\right)=0$ for $u>0$ and $j \neq \operatorname{deg}\left(h_{L}\right)=\operatorname{dim} P_{L}=\operatorname{dim} X^{\prime}$ (combine Serre duality from [Ful93, $\S 4.4$ ] with [Mus02, Theorem 3.4]). In particular, we have $H^{i}\left(X, L^{\operatorname{deg}\left(h_{L}\right)-r(L)-i}\right)=0$ for $i \neq \operatorname{deg}\left(h_{L}\right)$. When $i=\operatorname{deg}\left(h_{L}\right)$, we also have $0=h_{L}(-r(L))=\chi\left(L^{-r(L)}\right)=(-1)^{i} \operatorname{dim} H^{i}\left(X, L^{\operatorname{deg}\left(h_{L}\right)-r(L)-i}\right)$.

Proof of Corollary 1.3. In light of Lemma 4.1, the claim is the special case of Theorem 1.1 with $\ell=1, B_{1}=L, \boldsymbol{w}_{1}=\max \left\{\operatorname{deg}\left(h_{L}\right)-r(L), 1\right\}$ and $\boldsymbol{w}_{i}=1$ for $i>1$.

Proof of Corollary 1.2. The case $p=0$ is in [EW91]; for $p \geqslant 1$, it follows from Corollary 1.3.

The following well-known examples illustrate that Corollary 1.3 is sharp in some cases.

Example 4.2. Let $L$ be the ample line bundle on the toric variety $X$ corresponding to the polytope $\operatorname{conv}\{(1,0),(0,1),(1,1),(2,2)\} \subset \mathbb{R}^{2}$. The homogeneous ideal of $X$ in $\mathbb{P}^{3}=\mathbb{P}\left(H^{0}(X, L)^{*}\right)$ is generated by the cubic $x_{2}^{3}-x_{0} x_{1} x_{3}$ which implies that $L$ does not satisfy $\left(N_{1}\right)$. Calculations in [GS06] show that $L^{2}$ satisfies $\left(N_{3}\right)$ but not $\left(N_{4}\right)$.

Example 4.3. Let $\boldsymbol{e}_{1}, \ldots, \boldsymbol{e}_{n}$ be the standard basis of $\mathbb{R}^{n}$ and let $L$ be the ample line bundle on the toric variety $X$ corresponding to $P=\operatorname{conv}\left\{\mathbf{0}, \boldsymbol{e}_{1}, \ldots, \boldsymbol{e}_{n-1}, \boldsymbol{e}_{1}+\cdots+\boldsymbol{e}_{n-1}+(n-1) \boldsymbol{e}_{n}\right\} \subset \mathbb{R}^{n}$. Since $X$ is $n$-dimensional and singular, the morphism $X \rightarrow \mathbb{P}^{n}=\mathbb{P}\left(H^{0}(X, L)^{*}\right)$ is obviously not an embedding. The natural map $S \rightarrow R$ is not surjective and $(n-2) P$ is not normal because $(1, \ldots, 1)$ lies in $2(n-2) P$ but cannot be written as an integral combination of two lattice points in $(n-2) P$. For $n=3$, calculations in [GS06] show that $L^{2}$ satisfies $\left(N_{1}\right)$ but not $\left(N_{2}\right)$. 


\section{Hering, H. Schenck And G. G. Smith}

Proof of Corollary 1.4. Given a lattice polytope $P$, let $X$ be the corresponding toric variety and $L$ the associated ample line bundle on $X$. Since $P$ is normal if and only if $L$ satisfies $\left(N_{0}\right)$, the result follows from Corollary 1.3 and the fact that $r(P)=r(L)$.

Proof of Corollary 1.5. Let $\pi_{i}: X \rightarrow \mathbb{P}^{n_{i}}$ be the $i$ th projection and set $B_{i}:=\pi_{i}^{*}\left(\mathscr{O}_{\mathbb{P}^{n_{i}}}(1)\right)$. If $I:=$ $\left\{i \in\{1, \ldots, \ell\}: d_{i} \neq 0\right\}$, then $\mathscr{O}_{X}\left(d_{1}, \ldots, d_{\ell}\right) \cong \bigotimes_{i \in I} B_{i}^{d_{i}}$. Let $d:=\min \left\{d_{i}-1: i \in I\right\}$ and let $\mathcal{B}$ be the semigroup generated by $\left\{B_{i}: i \in I\right\}$. Proposition 6.10 in [MS04] proves that $\mathscr{O}_{X}$ is $\mathscr{O}_{X}$-regular with respect to $B_{1}, \ldots, B_{\ell}$. Thus, Theorem 2.1 shows that $\bigotimes_{i \in I} B_{i}^{d_{i}-d}$ is $\mathscr{O}_{X}$-regular with respect to $\left\{B_{i}: i \in I\right\}$ and lies in $\bigcap_{i \in I}\left(B_{i} \otimes \mathcal{B}\right)$. Since $\bigotimes_{i \in I} B_{i} \in \bigcap_{j \in I}\left(B_{j} \otimes \mathcal{B}\right)$, Theorem 1.1 applies with $\boldsymbol{w}_{1}=\left(d_{1}-d, \ldots, d_{\ell}-d\right)$ and $\boldsymbol{w}_{j}=(1, \ldots, 1)$ for $j \geqslant 2$.

Now assume that $B_{1}, \ldots, B_{\ell}$ are the minimal generators of $\operatorname{Nef}(X)$. To apply our techniques to adjoint bundles, we need to find $\boldsymbol{u}$ with $K_{X} \otimes B^{\boldsymbol{u}} \in \mathcal{B}=\operatorname{Nef}(X)$. Inspired by Fujita's conjectures, Corollary 0.2 in [Fuj03] provides the following necessary criterion: Let $X$ be a projective toric variety (not isomorphic to $\mathbb{P}^{n}$ ) such that the canonical divisor $K_{X}$ is $\mathbb{Q}$-Cartier. If $D$ is a $\mathbb{Q}$-Cartier divisor such that $D \cdot C \geqslant n$ for all torus invariant curves $C$, then $K_{X}+D$ is nef.

Proof of Corollary 1.6. If $X=\mathbb{P}^{n}$, then $K_{X}=\mathscr{O}_{X}(-n-1)$; Corollary 1.3 proves that $K_{X} \otimes B^{m_{n+1+p}}$ satisfies $\left(N_{p}\right)$. For general $X$, Theorem 3.4 in [Mus02] shows that $K_{X} \otimes B^{\boldsymbol{m}_{n+1}}$ is $\mathscr{O}_{X}$-regular with respect to $B_{1}, \ldots, B_{\ell}$. For any torus invariant curve $C$, there is a $B_{i}$ such that $B_{i} \cdot C>0$. Since $B^{\boldsymbol{m}_{n}}=B_{i}^{n} \otimes B^{\prime}$ where $B^{\prime}$ is globally generated, Corollary 0.2 in [Fuj03] implies that $K_{X} \otimes B^{\boldsymbol{m}_{n}} \in \mathcal{B}$. It follows that $K_{X} \otimes B^{\boldsymbol{m}_{n+1}} \in \bigcap_{j=1}^{\ell}\left(B_{j} \otimes \mathcal{B}\right)$ and Theorem 1.1 proves the claim.

The singular cubic surface in Example 4.2 also demonstrates that Corollary 1.6 can be sharp; see [GP99] for more examples of this type.

Example 4.4. Let $(X, L)$ be the normal cubic surface and ample line bundle defined in Example 4.2. It follows that $K_{X}^{-1}=L$ and $L$ is the minimal generator of $\operatorname{Nef}(X)$. Example 4.2 shows that $K_{X} \otimes L^{2}=L$ does not satisfy $\left(N_{1}\right)$. Hence, Corollary 1.6 provides the smallest $m \in \mathbb{N}$ (namely $m=3)$ such that $K_{X} \otimes L^{m}$ satisfies $\left(N_{1}\right)$.

For toric surfaces, it follows from [Sch04] that all of our corollaries are not optimal for $p \geqslant 2$. Specifically, given an ample line bundle $L \nsubseteq \mathscr{O}_{\mathbb{P}^{2}}(1)$ on a Gorenstein toric surface $X, K_{X} \otimes L^{m}$ satisfies $N_{3(m-2)}$ for $m \geqslant 2$ and $m \neq 4$, and $K_{X} \otimes L^{4}$ satisfies $N_{5}$.

\section{ACKNOWLEDGEMENTS}

We thank A. Bayer, W. Fulton, B. Harbourne, R. Lazarsfeld, M. Mustaţă and S. Payne for helpful discussions. Parts of this work were done while the last two authors were visiting the Mathematical Sciences Research Institute in Berkeley and the Mathematisches Forschungsinstitut in Oberwolfach. The second author was partially supported by NSF Grant DMS 03-11142 and the third author was partially supported by NSERC.

\section{REFERENCES}

BB96 V. Batyrev and L. Borisov, On Calabi-Yau complete intersections in toric varieties, in Higherdimensional complex varieties, Trento, 1994 (de Gruyter, Berlin, 1996), 39-65.

Bou80 N. Bourbaki, Élements de mathématique. Algèbre, Chapitre 10, Algèbre homologique (Masson, Paris, 1980).

BGT97 W. Bruns, J. Gubeladze and N. V. Trung, Normal polytopes, triangulations, and Koszul algebras, J. reine angew. Math. 485 (1997), 123-160. 


\section{SyZYGIES, MULTIGRADED REGULARITY AND TORIC VARIETIES}

But94 D. Butler, Normal generation of vector bundles over a curve, J. Differential Geom. 39 (1994), $1-34$.

EL93 L. Ein and R. Lazarsfeld, Syzygies and Koszul cohomology of smooth projective varieties of arbitrary dimension, Invent. Math. 111 (1993), 51-67.

EGHP06 D. Eisenbud, M. L. Green, K. Hulek and S. Popescu, Restricting linear syzygies: algebra and geometry, Compositio Math. 141 (2006), 1460-1478.

EW91 G. Ewald and U. Wessels, On the ampleness of invertible sheaves in complete projective toric varieties, Results Math. 19 (1991), 275-278.

Fak02 N. Fakhruddin, Multiplication maps of linear systems on smooth projective toric surfaces, Preprint (2002), math.AG/0208178.

Fuj03 O. Fujino, Notes on toric varieties from Mori theoretic viewpoint, Tohoku Math. J. (2) 55 (2003), $551-564$.

Ful93 W. Fulton, Introduction to toric varieties, Annals of Mathematics Studies, vol. 131 (Princeton University Press, Princeton, NJ, 1993).

GP99 F. J. Gallego and B. P. Purnaprajna, Projective normality and syzygies of algebraic surfaces, J. reine angew. Math. 506 (1999), 145-180.

GP01 F. J. Gallego and B. P. Purnaprajna, Some results on rational surfaces and Fano varieties, J. reine angew. Math. 538 (2001), 25-55.

GS06 D. R. Grayson and M. E. Stillman, Macaulay 2, a software system for research in algebraic geometry, version 0.9.2, 2006, available at http://www.math.uiuc.edu/Macaulay2/.

Gre84a M. L. Green, Koszul cohomology and the geometry of projective varieties, J. Differential Geom. 19 (1984), 125-171.

Gre84b M. L. Green, Koszul cohomology and the geometry of projective varieties II, J. Differential Geom. 20 (1984), 279-289.

GL85 M. L. Green and R. Lazarsfeld, On the projective normality of complete linear series on an algebraic curve, Invent. Math. 83 (1985), 73-90.

GL88 M. L. Green and R. Lazarsfeld, Some results on syzygies of finite sets and algebraic curves, Compositio Math. 67 (1988), 301-314.

Har97 B. Harbourne, Birational morphisms of rational surfaces, J. Algebra 190 (1997), 145-162.

Kle66 S. L. Kleiman, Toward a numerical theory of ampleness, Ann. of Math. (2) 84 (1966), 293-344.

Koe93 R. Koelman, A criterion for the ideal of a projectively embedded toric surface to be generated by quadrics, Beiträge Algebra Geom. 34 (1993), 57-62.

Las78 A. Lascoux, Syzygies des variétés déterminantales, Adv. Math. 30 (1978), 202-237.

Laz89 R. Lazarsfeld, A sampling of vector bundle techniques in the study of linear series, in Lectures on Riemann surfaces (World Scientific, Teaneck, NJ, 1989), 500-559.

Laz04 R. Lazarsfeld, Positivity in algebraic geometry I, Ergebnisse der Mathematik und ihrer Grenzgebiete (3), vol. 48 (Springer, Berlin, 2004).

LTZ93 J. Y. Liu, L. E. Trotter Jr and G. M. Ziegler, On the height of the minimal Hilbert basis, Results Math. 23 (1993), 374-376.

MS04 D. Maclagan and G. G. Smith, Multigraded Castelnuovo-Mumford regularity, J. reine angew. Math. 571 (2004), 179-212.

Mum66 D. Mumford, Lectures on curves on an algebraic surface, Annals of Mathematics Studies, vol. 59 (Princeton University Press, Princeton, NJ, 1966).

Mum70 D. Mumford, Varieties defined by quadratic equations, in Questions on algebraic varieties, CIME, III Ciclo, Varenna, 1969 (Edizioni Cremonese, Rome, 1970), 29-100.

Mus02 M. Mustaţă, Vanishing theorems on toric varieties, Tohoku Math. J. (2) 54 (2002), 451-470.

Oga03 S. Ogata, On quadratic generation of ideals defining projective toric varieties, Kodai Math. J. 26 (2003), 137-146.

Oga04 S. Ogata, On higher syzygies of projective toric varieties, Preprint (2004). 


\section{SyZygies, MULTigRADED REgULARITY AND TORIC VARIETIES}

ON02 S. Ogata and K. Nakagawa, On generators of ideals defining projective toric varieties, Manuscripta Math. 108 (2002), 33-42.

OP01 G. Ottaviani and R. Paoletti, Syzygies of Veronese embeddings, Compositio Math. 125 (2001), 31-37.

Par00 G. Pareschi, Syzygies of abelian varieties, J. Amer. Math. Soc. 13 (2000), 651-664.

PP04 G. Parsechi and M. Popa, Regularity on abelian varieties. II. Basic results on linear series and defining equations, J. Algebraic Geom. 13 (2004), 167-193.

PW85 P. Pragacz and J. Weyman, Complexes associated with trace and evaluation. Another approach to Lascoux's resolution, Adv. Math. 57 (1985), 163-207.

Rub02 E. Rubei, On syzygies of Segre embeddings, Proc. Amer. Math. Soc. 130 (2002), 3483-3493.

Rub04 E. Rubei, Resolutions of Segre embeddings of projective spaces of any dimension, Preprint (2004), math.AG/0404417, J. Pure Appl. Algebra, to appear.

Sch04 H. Schenck, Lattice polygons and Green's theorem, Proc. Amer. Math. Soc. 132 (2004), 3509-3512.

Sta97 R. P. Stanley, Enumerative combinatorics. Vol. 1, Cambridge Studies in Advanced Mathematics, vol. 49 (Cambridge University Press, Cambridge, 1997).

Milena Hering mhering@umich.edu

Department of Mathematics, University of Michigan, Ann Arbor, MI 48109, USA

Hal Schenck schenck@math.tamu.edu

Mathematics Department, Texas A\&M University, College Station, TX 77843, USA

Gregory G. Smith ggsmith@mast.queensu.ca

Department of Mathematics and Statistics, Queen's University, Kingston, Ontario K7L 3N6, Canada 